\title{
Determining Class Proportions Within a Pixel Using a New Mixed-Label Analysis Method
}

\author{
Xiaoping Liu, Xia Li, and Xiaohu Zhang
}

\begin{abstract}
Land-cover classification is perhaps one of the most important applications of remote-sensing data. There are limitations with conventional (hard) classification methods because mixed pixels are often abundant in remote-sensing images, and they cannot be appropriately or accurately classified by these methods. This paper presents a new approach in improving the classification performance of remote-sensing applications based on mixed-label analysis (MLA). This MLA model can determine class proportions within a pixel in producing soft classification from remote-sensing data. Simulated images and real data sets are used to illustrate the simplicity and effectiveness of this proposed approach. Classification accuracy achieved by MLA is compared with other conventional methods such as linear spectral mixture models, maximum likelihood, minimum distance, and artificial neural networks. Experiments have demonstrated that this new method can generate more accurate land-cover maps, even in the presence of uncertainties in the form of mixed pixels.
\end{abstract}

Index Terms-mixed pixels, Mixed-label analysis (MLA), remote sensing, soft classification.

\section{INTRODUCTION}

$\mathbf{R}$ EMOTE SENSING has become an important source of land use/cover information at a range of spatial and temporal scales [1]. In the last decade, a variety of classification algorithms, both parametric and nonparametric, has been developed to classify remote-sensing data. These methods include statistical classifiers [2], knowledge-based systems [3], neural networks [4], and swarm intelligence [5]. One major limitation of these per-pixel classifiers is that they were developed for the classification of classes that is considered to be discrete and mutually exclusive and rely on the assumption that each pixel is pure [6]. However, such assumptions are often invalid in areas where the classes exist as continua rather than as a mosaic of discrete classes. In fact, remote-sensing images, particularly at coarse spatial resolutions, are commonly dominated by mixed pixels that contain more than one class on the ground [7]. For

Manuscript received March 3, 2009; revised June 1, 2009. First published November 13, 2009; current version published March 24, 2010. This work was supported in part by the National Natural Science Foundation of China under Grant 40901187, by the Key National Natural Science Foundation of China under Grant 40830532, by the National Outstanding Youth Foundation of China under Grant 40525002, by the Guangdong Provincial Natural Science Foundation of China under Grant 9451027501002471, and by the Research Fund of LREIS, CAS, under Grant 4106298.

$\mathrm{X}$. Liu and X. Li are with the School of Geography and Planning, Sun Yat-sen University, Guangzhou 510275, China (e-mail: yiernanh@163.com; lixia@mail.sysu.edu.cn).

X. Zhang was with the School of Geography and Planning, Sun Yat-sen University, Guangzhou 510275, China. He is now with the University of Hong Kong, Hong Kong (e-mail: xiaohu.zhang.cn@gmail.com).

Color versions of one or more of the figures in this paper are available online at http://ieeexplore.iee.org.

Digital Object Identifier 10.1109/TGRS.2009.2033178 instance, mixed pixels will occur frequently in the interclass transition zones. One single pixel may contain more than one type of geographical object such as trees, grass, water, and soil. Mixed pixels occur because the pixel size may not be fine enough to capture the details on the ground necessary for specific applications [8]. They may also occur where the ground properties such as vegetation and soil types vary continuously [9]. These mixed pixels reflect the composite spectral response of the classes within them [10]. Application of perpixel approaches to images dominated with mixed pixels may result in an erroneous classification. Much attention has been directed at the development of alternative algorithms to solution of the mixed-pixel problem in mapping land cover from remotesensing images [11], [12]. Alternative algorithms such as linear mixture modeling and fuzzy or soft classification are mainly focused on the derivation of estimates of the subpixel class composition.

The linear spectral mixture model (LSMM) has been widely used for classification of remote-sensing data to produce soft classification outputs. It estimates the abundance fractions of spectral signatures within mixed pixels [13]-[15]. However, LSMM is based on an unattainable assumption that class mixing is performed in a linear manner and adopts a least square procedure to estimate the class proportions within each pixel [16]. Moreover, the LSMM approach cannot satisfy the requirement that each class proportion in the analysis result is within the range $(0-1)$. Another commonly used approach in estimating the class composition of pixels for land-cover mapping applications is the use of a fuzzy classification, which allows for the multiple and partial class membership properties of mixed pixels [17]. It has been found that a fuzzy classification can provide more informative and potentially more accurate representations of land cover than conventional classification [18]. The output of such approaches is typically a set of fraction images, each of which describes some measure of the fractional cover of a particular land-cover class within each pixel. The principal limitations associated with fuzzy approaches include the inherent subjectivity in the derivation of the membership functions needed to guide classification [19].

Recently, multilabel learning algorithms were proposed to solve mixed-label problems [20], [21]. In contrast to conventional data classification, where each instance is assigned to only one label, for multilabel classification, one instance may be simultaneously relevant to several labels. This approach has a potential in solving mixed-pixel problems in remote-sensing classification. Unfortunately, multilabel classification approach is only capable of identifying the classes of a mixed-label data but cannot give the class proportions within each pixel. 


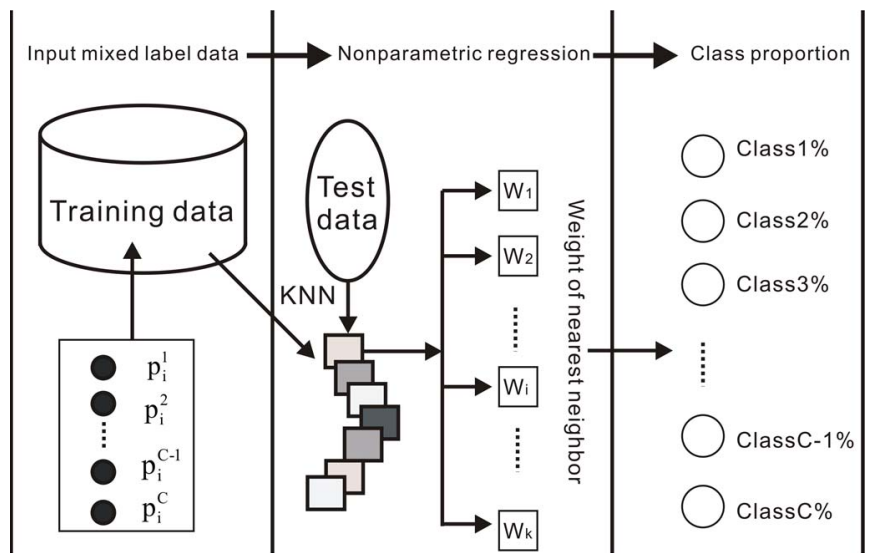

Fig. 1. MLA model based on the nonparametric regression algorithm.

This paper proposes a new mixed-label analysis (MLA) model based on nonparametric regression. The proposed model mainly aims to solve two problems. First of all, MLA is used to determine the classes for mixed-label data, which is a question which multilabel learning approach can also answer in a general sense. Second, MLA will play an important role in acquiring class proportions in mixed-label data. The proposed model should overcome the limitations of conventional classification, thus becoming an important tool in classifying remote-sensing images which are dominated by mixed pixels. Simulated image and real data sets will be used to illustrate the simplicity and effectiveness of this proposed approach. The remainder of this paper is organized as follows. A detailed description of MLA to an analysis of mixed-label data is given in Section II. The experimental results when using this proposed approach to produce a soft classification are provided in Section III. Finally, the discussion of the results and the concluding remarks are presented in Section IV.

\section{MLA MODEL}

Let $T=\left\{\left(x_{1}, Y_{1}\right), \ldots,\left(x_{i}, Y_{i}\right), \ldots,\left(x_{m}, Y_{m}\right)\right\}\left(x_{i} \in X, Y_{i} \in\right.$ $Y$ ) be a sequence of training examples, where $x_{i}$ belongs to a domain or instance space $X$ and $Y$ is the output space. Each instance has $n$ attributes [i.e., $\left.x_{i}=\left(a_{i}^{1}, \ldots, a_{i}^{n}\right)\right]$. The total number of classes (labels) for the training data set is $C ; Y_{i}$ refers to the class proportions [i.e., $Y_{i}=\left(p_{i}^{1}, \ldots, p_{i}^{C}\right)$, $\sum_{c=1}^{C} p_{i}^{C}=1$, where $p_{i}^{C}$ refers to the $c$ th class proportion in the $i$ th sample].

The task of the MLA model is to find a function $f(x)$, which takes as input an instance $x_{i}$ and return the class proportions in the mixed-label data. In this paper, the $k$-nearest neighbor (k-NN) nonparametric regression algorithm is used to construct this MLA model, and the structure of MLA is shown in Fig. 1.

Numerous forecasting techniques belong to parametric algorithms, which assume that the data to be modeled take on a structure that can be described by a known mathematical expression with a few free parameters [22]. Nonparametric regression is an alternative approach that does not make any rigid assumptions about the data. It is a forecasting technique similar to case-based reasoning that relies on the data to determine a relationship between input and output states [23]. One of these techniques is the k-NN nonparametric regression, which has been widely applied in pattern recognition and statistical classification tasks [24].

This proposed MLA is established by using nonparametric regression as follows:

$$
\hat{Y}=f\left(x_{i}\right)+u_{i}, \quad i=1, \ldots, m
$$

where $f(x)=E(Y \mid X=x)$ is a regression function for $Y$ to $X$ and $u_{i}$ is a stochastic error item. The nonparametric regression model can give an estimate value $\hat{f}_{m}(x)$ of the regression function $f(x)$ for a given sample $T=$ $\left\{\left(x_{1}, Y_{1}\right), \ldots,\left(x_{i}, Y_{i}\right) \ldots,\left(x_{m}, Y_{m}\right)\right\}\left(x_{i} \in X, Y_{i} \in Y\right)$; here, $\hat{f}_{m}(x)$ is generally estimated by using the following:

$$
\hat{Y}=\hat{f}_{m}(x)=\sum_{i=1}^{m} W_{i}\left(x, x_{1}, x_{2}, \ldots, x_{m}\right) Y_{i}
$$

where $W_{i}\left(x, x_{1}, x_{2}, \ldots, x_{m}\right)$ is a weight function, which is a measure of the influence or contribution of the sample $\left(x_{i}, Y_{i}\right)$ in estimating $\hat{f}_{m}(x)$. The weight function shall satisfy the following condition:

$$
W_{i}\left(x, x_{1}, x_{2}, \ldots, x_{m}\right) \geq 0 \quad \sum_{i=1}^{m} W_{i}\left(x, x_{1}, x_{2}, \ldots, x_{m}\right)=1 .
$$

The k-NN method is used to determine the weights of MLA. The algorithm assumes that the two instances with the smaller distance are recognized as similar ones, which are correspondingly taken as the same class ( $k$ neighbors) and as the best match in the training instances. In most classical k-NN variants, distance measure is calculated by using the Euclidean distance

$$
d_{i}=d\left(x, x_{i}\right)=\sqrt{\sum_{q=1}^{n}\left(a_{i}^{q}-a^{q}\right)^{2}}
$$

where $a_{i}^{q}$ and $a^{q}$ are the $q$ th features of each vectors and $n$ is the number of attribute variables. In using a weight function to estimate $\hat{f}_{m}(x)$, only the influences of k-NNs will be taken into account. With regard to this k-NN approach, the key lies in determining the contribution from each nearest neighbor. Generally, the reciprocal of the distance $\left(1 / d_{i}\right)$ is used to calculate the weight of each k-NN [25]

$$
W_{i}\left(x, x_{1}, x_{2}, \ldots, x_{k}\right)=\frac{1 / d_{i}}{\sum_{j=1}^{k} 1 / d_{j}} .
$$

This approach appears to be too simplistic as it assumes a linear relationship between the weight of the nearest neighbor and the reciprocal of its distance. Here, a negative-index (NI) approach is proposed to calculate the weights of k-NNs

$$
W_{i}\left(x, x_{1}, x_{2}, \ldots, x_{k}\right)=\frac{a+e^{-c d_{i}}}{\sum_{j=1}^{k}\left(a+e^{-c d_{j}}\right)}
$$




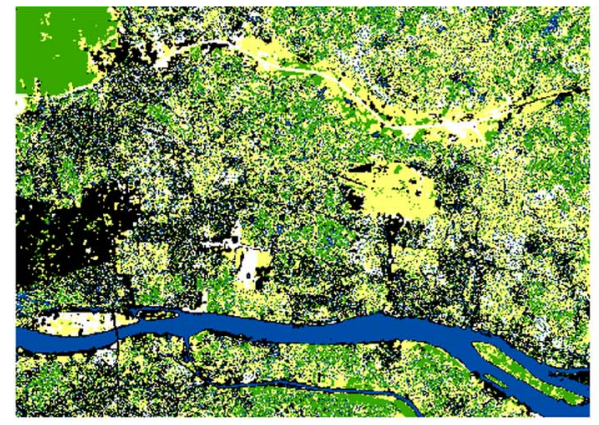

(a)

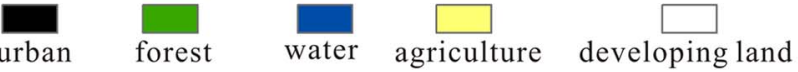

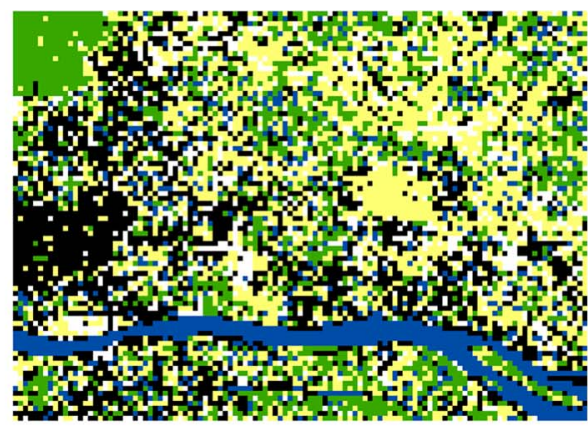

(b)

Fig. 2. Simulated image showing urban, forest, water, agriculture, and developing land.

where values for parameters $a$ and $c$ should be determined. By gradually changing both of the aforementioned parameters $(a, c)$ in the algorithm and evaluating root mean squared residual (RMSE) for MLA, the optimal parameter settings can be searched. The detailed optimization progress will be provided in the next section.

\section{Application of MLA in Simulated AND REMOTE-SENSING DATA}

MLA is used to analyze or classify two data sets, simulated images, and real remote-sensing images. Application to a simulated data set allows us to examine this method in a controlled manner, as the actual outputs are known a priori.

\section{A. Simulated Data}

The simulated data are preferred in validating MLA because the actual class proportions of each pixel in the image are known beforehand. These data are mainly used to verify the analysis capability of the MLA model in different amounts of noise environment. In this experiment, the simulated data correspond to $500 \times 350$ pixel data in five bands comprised of five assumed land-use classes (urban, forest, water, agriculture, and developing land) and have digital number (DN) values ranging from 10 to 255. Urban appears in black, forest appears in green, water appears in blue, agriculture appears in yellow, and developing land appears in white [Fig. 2(a)]. Then, spatial degradation was done by simple averaging of $\mathrm{DN}$ values of $4 \times 4$ pixels in the original simulated image, following the procedure in Maselli et al. [26]. The degraded image, which is used for the analyses, is shown in Fig. 2(b). Finally, the proportions of the classes were computed in the degraded image by simply considering the number of the classes contained in each degraded pixel based on the original simulated data. The individual class proportion images are also known as fraction images [Fig. 5(a)]. These images represent actual class proportions and provide the reference data. However, it should be noted that this method of synthesizing data sets is without regard to the so-called point spread function of imaging devices [27], and the properties of the degraded data were not equal to those of the data originally acquired at a coarser spatial resolution [26]. This method of computation of the class proportions, although not rigorous, is considered adequate for the illustrations in our experiment.

Generally, the training and testing sample sizes are selected arbitrarily [16], [28]. It remains a question whether the estimation accuracy would be significantly changed if a different size of training data is used. This may result in a suboptimal estimation result when the size of the training data is too small, whereas it takes a long computation when the size chosen is too large. We examined the effect of the number of training data using 12 reference data set sizes. The size of the training data is varied between 100 and 1200, with increments of 100 . A total of 1500 samples were randomly selected as the testing data. The training data take the form of $T_{\text {training }}=\left\{\left(\mathbf{B}_{\mathbf{i}}, \mathbf{P}_{\mathbf{i}}\right) \mid i=\right.$ $1,2, \ldots, m\}$, where $\mathbf{B}_{\mathbf{i}}=\left(b_{i}^{1}, b_{i}^{2}, b_{i}^{3}, b_{i}^{4}, b_{i}^{5}\right)$ is the DN value vector and $\mathbf{P}_{\mathbf{i}}$ is the class proportion vector.

In the k-NN nonparametric regression algorithm, the reciprocal distance $(\mathrm{RD})$ is generally used to determine the weight of each nearest neighbor, but this weight-determining approach appears to be too simplistic. In this paper, we present an NI approach to modify the contribution of each nearest neighbor (weight) based on the determination of two parameters $a$ and $c$ [refer to (6)]. Here, $a$ and $c$ directly influence the results of the calculation of the weight $W_{i}$ and will ultimately affect the precision of the analysis using MLA. As a result, the key lies in how to optimize the parameters $(a, c)$ in order to improve the precision of MLA. To search the optimal parameter settings, we will gradually modify the values of the parameters $(a, c)$ and calculate root mean squared residual (RMSE) of MLA. RMSE is a quadratic scoring rule which measures the average magnitude of the error, and it acts as the performance measure of classification models in this experiment. When RMSE reaches its minimum, the values of $a$ and $c$ are selected as the optimal parameter settings for MLA. RMSE of the estimations is calculated as

$$
R M S E=\sqrt{(1 / N) \sum_{i=1}^{N}\left(p_{i}-\hat{p}_{i}\right)^{2}}
$$

where $N$ is the number of estimated and measured values and $p_{i}$ and $\hat{p}$ are the measured and estimated class proportions, respectively. In MLA, the precisions of multiple classes are 


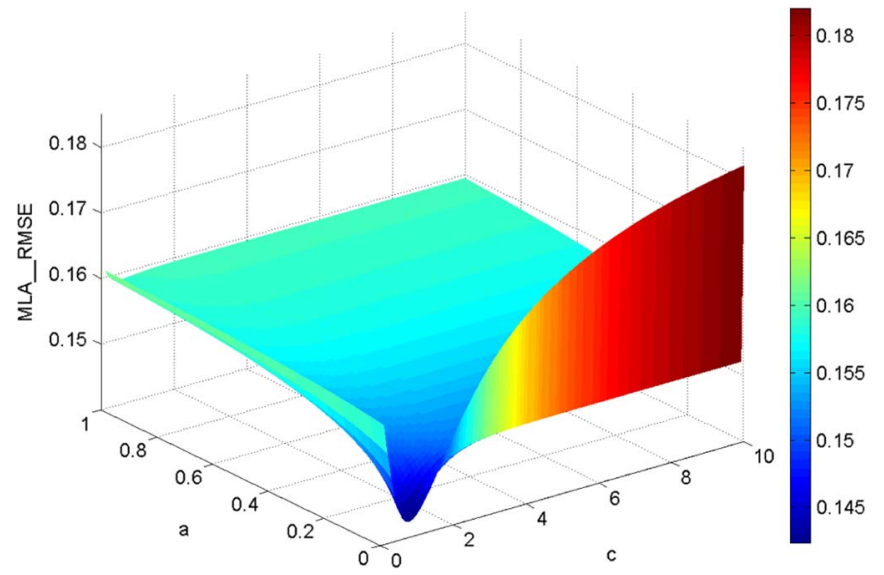

Fig. 3. MLA_RMSE values obtained using different combinations of parameters $(a, c)$ settings (simulated data).

required to be evaluated. Hence, the sum of the RMSE values of all classes is calculated as the performance measure of MLA

$$
M L A \_R M S E=\sum_{N C=1}^{C} R M S E_{N C}
$$

where $C$ denotes the number of classes and $R M S E_{N C}$ is the RMSE values of the $N C$ th class, which can be calculated by (7).

A greedy search strategy is used to search for the optimal parameter settings. We assigned values to parameter $a$ from 0 to 1 , with increments of 0.01. Parameter $c$ has been varied between 0.1 and 10, with increments of 0.1 . Then, MLA_RMSE is calculated for MLA under different combinations of $(a, c)$. When MLA_RMSE reaches its minimum, the values of $a$ and $c$ are considered as the optimal parameter settings. Five retrieved neighbors are used to avoid too much computation time in the search procedure. Fig. 3 shows the MLA_RMSE values obtained using the different combinations of $a$ and $c$ values with the 800-sample training data set. As shown in Fig. 3, when $a>0.5$, the technique does not seem to be sensitive to the choice of $a$; differences along parameter $a$ are relatively insignificant. It is not very sensitive to the choice of $c$ either, as long as $c>2$ in this case. However, when $a$ and $c$ both take small values (i.e., $a$ varies between 0 and 0.5 and $c$ varies between 0 and 2), it is very sensitive to the chosen parameters $a$ or $c$. MLA_RMSE is distributed spatially in the form of a tundish, which corresponds to the area for low MLA_RMSE values as shown in Fig. 3. The search for the optimal parameter settings continues until the minimum value of MLA_RMSE is found. The minimum value for MLA_RMSE was 0.1521 when $a=0.02$ and $c=0.74$.

The RMSE and classification efficiencies obtained by using different sizes of training data are shown in Figs. 4 and 5, which indicate that the increase in training data size can improve prediction accuracy but increase the computation time. However, further accuracy improvement is not obvious when the size of training data is greater than 800 .

In order to decrease the computation complexity and improve the accuracy of estimation, we selected 800 samples as the training data size to obtain the optimal parameter settings, and these parameter settings $(a=0.02$ and $c=0.74)$ were

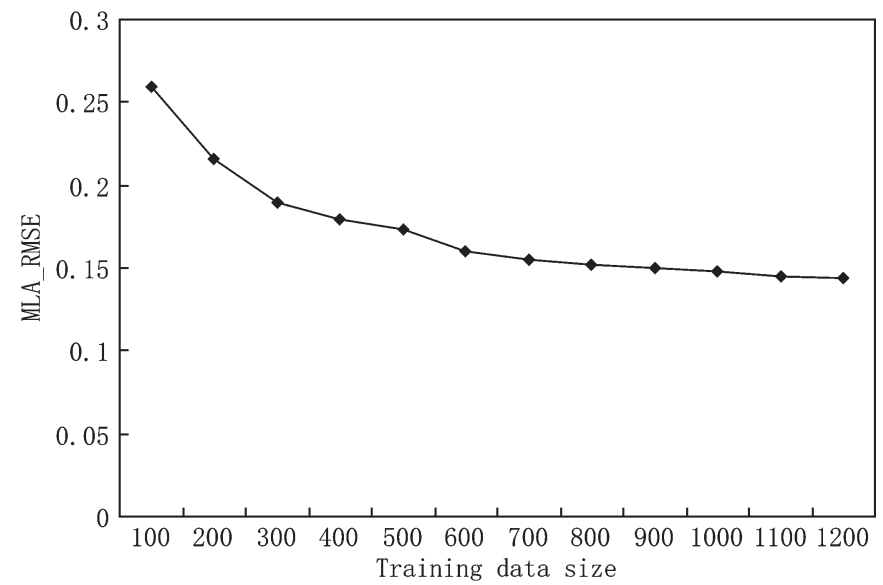

Fig. 4. Influence of training data size on the estimation accuracy of MLA (simulated data).

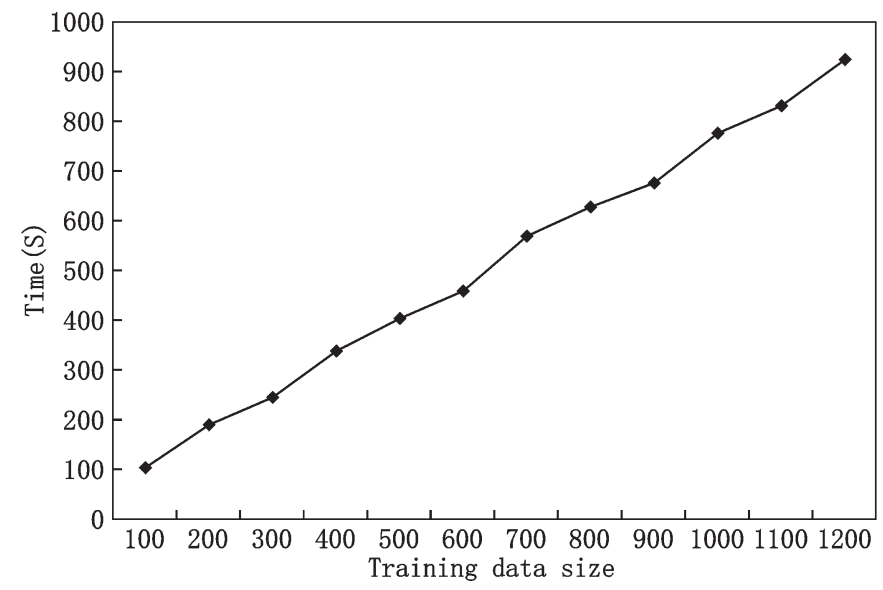

Fig. 5. Influence of training data size on the computation time of MLA (simulated data).

then used in classifying the simulated data using MLA. The class proportions were produced by this model [Fig. 6(b)]. Meanwhile, RMSE values of the test data were calculated. As shown in Table I, the values of the RMSE for urban, forest, water, agriculture, and developing land are 0.0321, 0.0206, $0.0319,0.0498$, and 0.0177 , respectively, indicating a very satisfactory classification accuracy for this MLA model. As shown in Fig. 6(c), the absolute residual value for a different land-use class appears to be black in tone as a whole, and the highest absolute residual does not exceed 0.2. The results indicate that the MLA model is highly capable of classifying simulated data in a noise-free environment.

The following experiment is designed to compare the sensitivity of the analysis results to the amount of noise present in the data. Different amounts of Gaussian white noise are added to the simulated image by using Matlab 7.1. The added noise is measured by the signal-to-noise ratio (often written as $S / N$ or SNR). SNR is a measure of signal strength relative to background noise, expressed in decibels, and is defined as follows:

$$
S N R=10 \log _{10} \frac{\operatorname{Var}(\text { image })}{\operatorname{Var}(\text { noise })}
$$




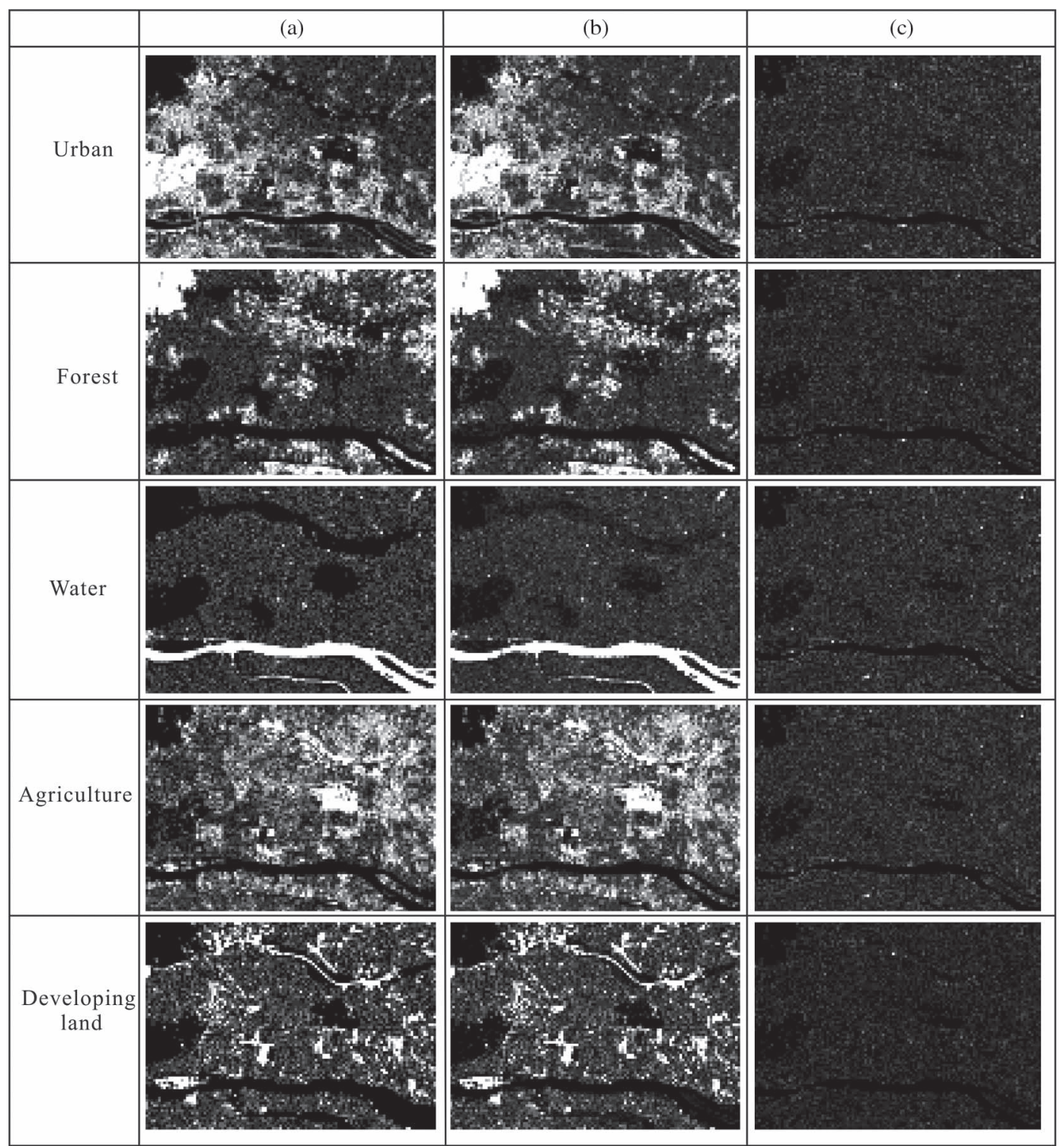

Fig. 6. Class proportion images and absolute residual for different land-use classes by using the MLA model (simulated data).

TABLE I

RMSE Values of Each Land-Use Class Using NI-MLA and RD-MLA (Simulated Data)

\begin{tabular}{lcccccc}
\hline Model & urban & forest & water & agriculture & developing land & MLA_RMSE \\
\hline NI-MLA & 0.0321 & 0.0206 & 0.0319 & 0.0498 & 0.0177 & 0.1521 \\
\hline RD-MLA & 0.0358 & 0.0231 & 0.0361 & 0.0525 & 0.0189 & 0.1664 \\
\hline
\end{tabular}

TABLE II

RMSE Values of Each Land-Use Class in Different Amounts of Noise EnVironment

\begin{tabular}{lcccccc}
\hline SNR & urban & forest & water & agriculture & developing land & MLA_RMSE \\
\hline $30 \mathrm{~dB}$ & 0.0451 & 0.0306 & 0.0425 & 0.0657 & 0.0286 & 0.2152 \\
$20 \mathrm{~dB}$ & 0.0812 & 0.0535 & 0.0619 & 0.0905 & 0.0487 & 0.3358 \\
$10 \mathrm{~dB}$ & 0.1409 & 0.1032 & 0.1096 & 0.1590 & 0.0950 & 0.6077 \\
$5 \mathrm{~dB}$ & 0.1932 & 0.1575 & 0.1648 & 0.2078 & 0.1511 & 0.8745 \\
$0 \mathrm{~dB}$ & 0.2483 & 0.2235 & 0.2260 & 0.2461 & 0.2137 & 1.4822 \\
\hline
\end{tabular}

In this experiment, the SNR is varied between $30 \mathrm{~dB}$ (slightly noisy) and $0 \mathrm{~dB}$ (extremely noisy). Estimation accuracies are shown in Table II, which shows that the increase in noise amount will decrease analysis accuracy. As shown in Table II, the values of MLA_RMSE for five SNR (30, 20, 10, 5, and 0) are $0.2152,0.3358,0.6077,0.8745$, and 1.4822 , respectively, 


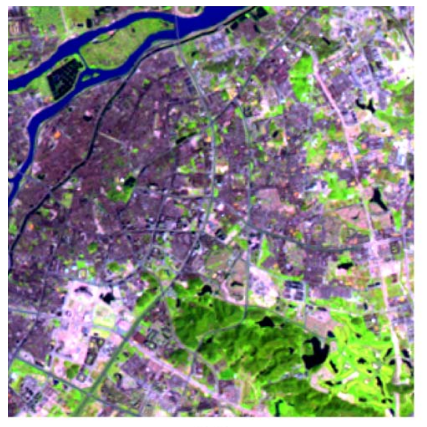

(a)

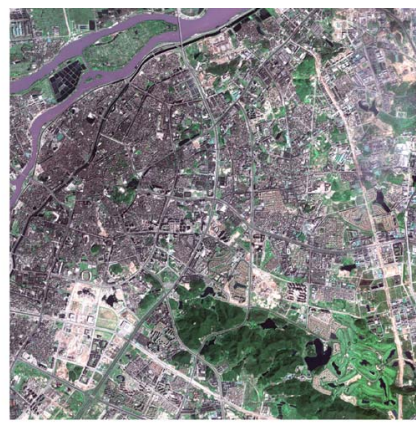

(b)
Fig. 7. TM images $(5,4,3)$ and QuickBird image in the study area.

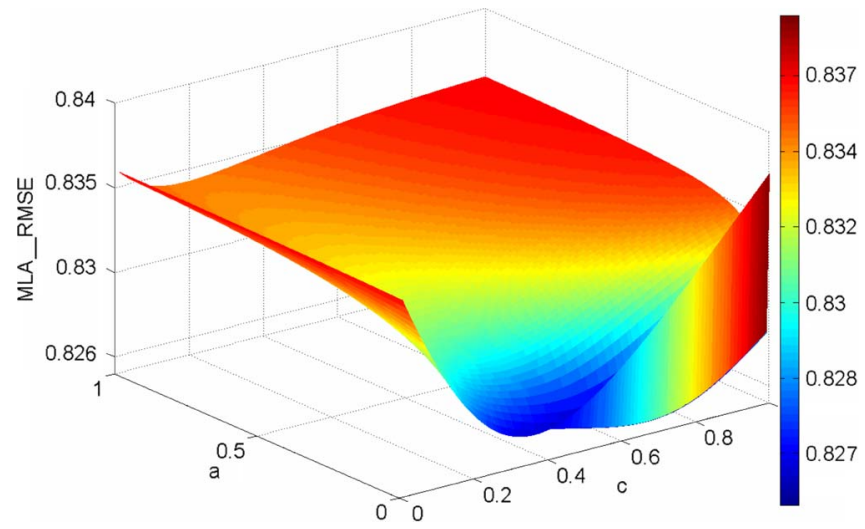

Fig. 8. MLA_RMSE values obtained using different combinations of parameters $(a, c)$ settings (TM image)

which indicates that the MLA model is capable of analyzing mixed-label data in different noise environment.

To establish a benchmark for the evaluation of the proposed NI-based MLA (NI-MLA) model, we compared it with the RD-based [refer to (5)] MLA (RD-MLA) model. As shown in Table I, the MLA_RMSE value of NI-MLA is smaller than that of RD-MLA. Therefore, this NI-MLA model shows higher analysis accuracy than the RD-MLA model.

\section{B. Actual Data From Remote-Sensing Images}

A satellite Landsat Thematic Mapper (TM) image of Dongguan in the Pearl River Delta acquired on July 18, 2003, was used to produce a land-use classification. The study area consists of $296 \times 299$ pixels, with a ground resolution of $30 \mathrm{~m}$ [Fig. 7(a)]. This area encompasses a diverse environment, which is dominated by the following six land-use types: residential, forest, water, cropland, grass, and developing land. A QuickBird image of $0.61-\mathrm{m}$ resolution in 2003 is used as reference data for the creation of training and testing data sets [Fig. 7(b)]. Because of the high resolution, each pixel in the image was assumed to be pure in terms of land-use classes. A total of 3000 samples were randomly selected from TM images, wherein 1937 had unique class labels and 1063 were mixed pixels. Then, class proportions within these samples were generated based on the visual interpretation of this QuickBird image and field investigation on the ground. A pixel of Landsat TM images corresponds to about $49 \times 49$ pixels of QuickBird

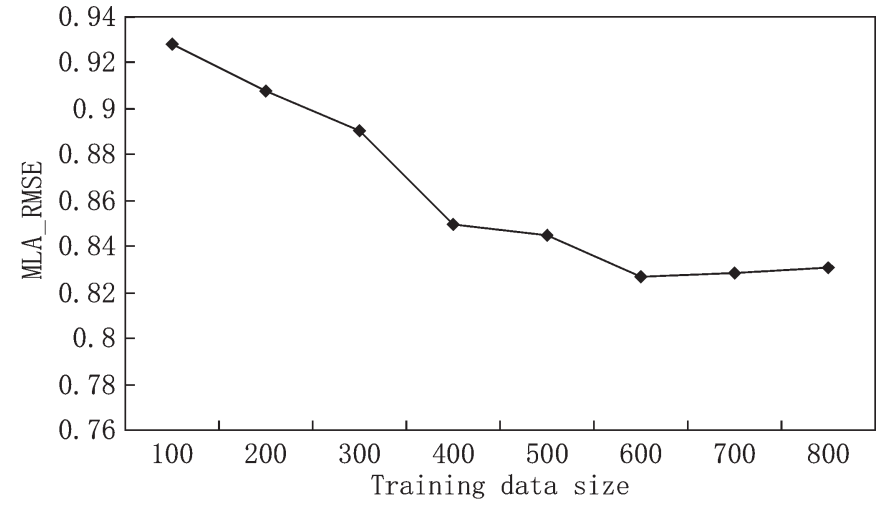

Fig. 9. Influence of training data size on the estimation accuracy of MLA (TM image).

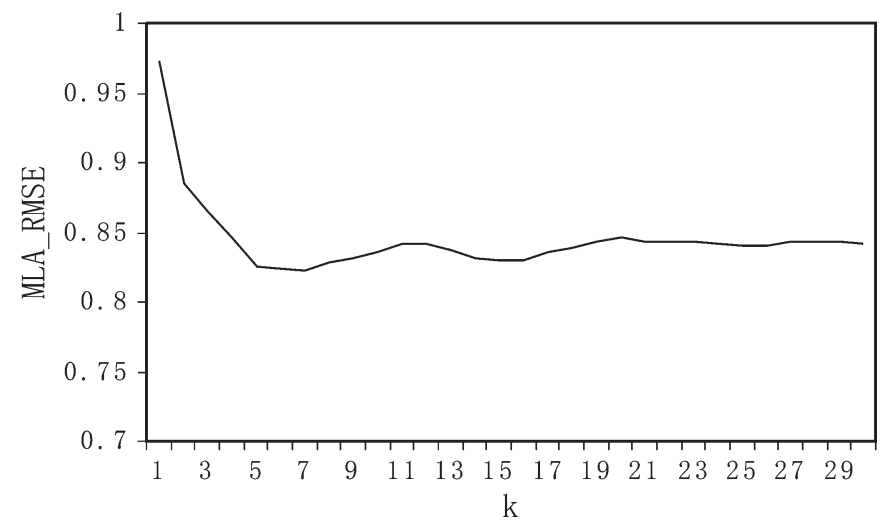

Fig. 10. Relationship between the number of $k$ and estimation accuracy.

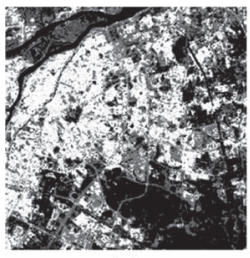

(a)

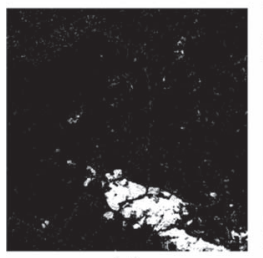

(d)

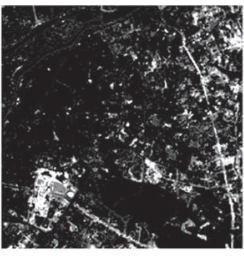

(b)

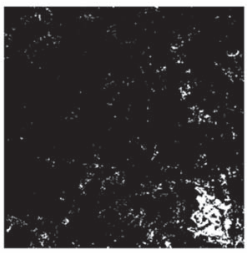

(e)

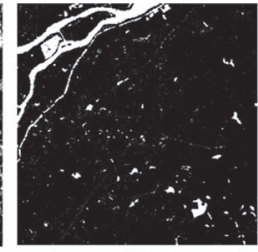

(c)

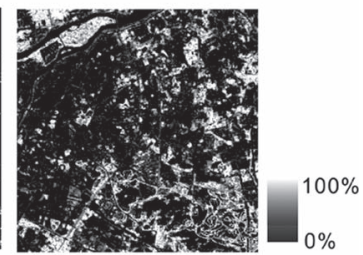

(f)
Fig. 11. Class proportion images for different land-use classes by using the MLA model (TM image).

images, so it is possible to acquire actual class proportions in each pixel of the TM image. These actual class proportions are used as reference data for training data and testing data collection. Samples for the training set are randomly chosen, and the size of the training data ranges from 100 to 800, with increments of 100. A total of 1800 samples were randomly selected as the testing data.

Compared with the simulated data, TM images were much more complicated in terms of land-use patterns and noise distribution. Based on the training set, the greedy search approach was used to determine the parameter settings $(a, c)$ in this MLA 
TABLE III

RMSE VALUES of EACH LAND-USE ClASS USING MLA AND LSMM (TM IMAGE)

\begin{tabular}{cccccccc}
\hline Model & urban & developing land & water & forest & grass & agriculture & MLA_RMSE \\
\hline MLA & 0.2034 & 0.1606 & 0.0969 & 0.0857 & 0.0762 & 0.2029 & 0.8257 \\
LSMM & 0.2349 & 0.1869 & 0.1135 & 0.2074 & 0.2669 & 0.3177 & 1.3273 \\
\hline
\end{tabular}

model. Parameters $a$ and $c$ were both assigned a value from 0 to 1 , with increments of 0.01 . The user of the $\mathrm{k}-\mathrm{NN}$ technique has to decide the number of nearest neighbors retrieved; five neighbors are selected in the search procedure. Fig. 8 shows the MLA_RMSE values obtained using the different combinations of $a$ and $c$ values with the 600-sample training data. The minimum value for MLA_RMSE was 0.8257 when $a=0.01$ and $c=0.67$.

As shown in Fig. 9, the larger the size of the training data is, the higher the accuracy of estimation becomes. However, there are slight differences in estimation accuracy when the training data size is greater than 600. Therefore, a total of 600 samples were selected as the data set size to obtain the optimal parameter settings.

Another parameter is the number of retrieved neighbors $(k)$. Experiments were carried out to test the influences of various $k$ values on the estimated results. Training sets of 600 samples were used to examine the relationships. The experiments indicate that the increase in $k$ values can improve the prediction accuracy but significantly increase the computation time. Fig. 10 shows the relationship between the number of $k$ and prediction accuracy based on the training data. The MLA_RMSE is 0.979 $(k=1), 0.8257(k=5), 0.8361(k=5)$, and $0.8460(k=20)$, respectively. It is obvious that the estimation accuracy becomes stabilized after $k$ becomes greater than five. Therefore, five neighbors are used to avoid too much computation time.

An experiment is designed to show the classification results of MLA when the training set contains only pure pixels. This experiment used 600 pixels for training, which are all pure. The value of MLA_RMSE for this pure training data is 0.8771 . The results demonstrate that the MLA model trained with examples of mixtures can have better estimation accuracy than pure training data.

These parameters ( $a=0.01, c=0.67$, and $k=5$ ) can yield the highest accuracy in classifying TM images. Therefore, these optimal parameter settings were used to classify the TM image. The class proportions were obtained by using this MLA model (Fig. 11). As can be found through a comparison between Figs. 7 and 11, this MLA model is capable of effectively separating various land-use classes and obtaining class proportions. The RMSE for the testing set is shown in Table III. Comparison between Tables I and III indicates that the classification accuracy of TM data is lower than that of the simulated data. This is mainly because the TM image data are subject to noise and a high spectral correlation between different landuse classes. However, the overall results of classifying TM data by this MLA model are satisfactory in terms of accuracies. Higher classification accuracies can be achieved for the land classes of water, forest, grass, and developing land. However, the classification accuracies for urban and agricultural classes are lower than those of other classes.

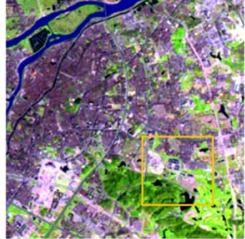

(a)

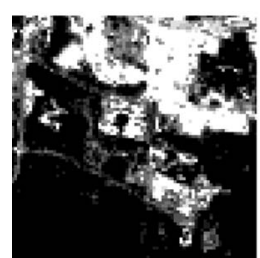

(d)

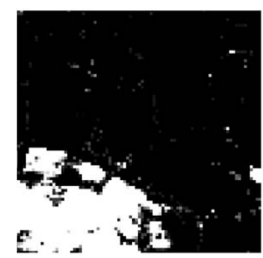

(g)

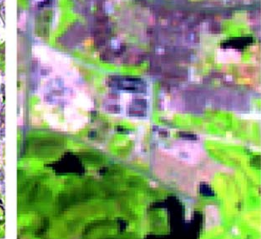

(b)

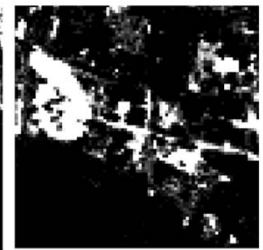

(e)

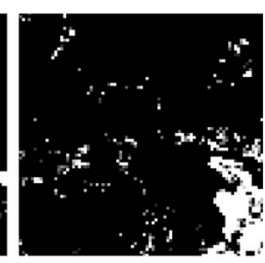

(h)

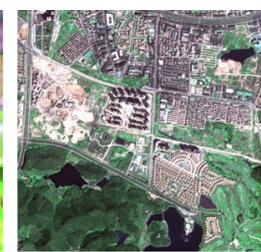

(c)

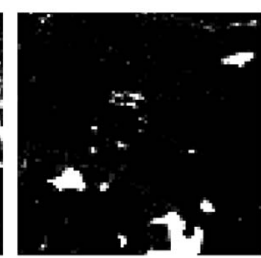

(f)

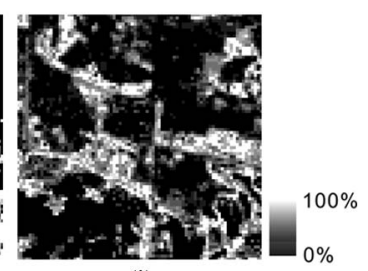

(i)
Fig. 12. Class proportions in the local enlargement area of Dongguan.

An enlarged part of the study area is shown in Fig. 12 This part consists of a quite diverse land-use types such as residential, forest, water, cropland, grass, and developing land. Visually comparing remote-sensing images [Fig. 12(b) and (c)] with fraction images can yield some interesting results. There are obvious changes in grayscale in the interclass transition zones. This indicates the area dominated by a large number of mixed pixels.

A further experiment is to compare the performances of this proposed model with those of the LSMM. LSMM has been widely used as a technique in analyzing the mixture of components in remotely sensed images [29], [30]. This method applies a linear model to estimate the abundance fractions of spectral signatures within mixed pixels [31]. Many endmember extraction algorithms have been developed to find endmembers, such as pixel purity index (PPI) [32], N-finder algorithm [33], iterative error analysis [34], minimum volume transform [35], convex cone analysis [36], and vertex component analysis [37]. In this paper, image endmembers are extracted by integrating QuickBird image and PPI, and pure pixels are selected manually by visualizing the PPI results in an N-dimensional visualizer and QuickBird image. Fig. 13 shows the class proportions by using the LSMM model. By comparing Fig. 13 with Fig. 12, we can find that the performance of this LSMM method is very poor in analyzing forest, grass, and cropland. The RMSE of these land-use classes are 0.2074, 0.2669, and 0.3177, respectively, (Table III), which were significantly worse than 


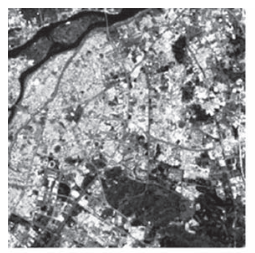

(a)

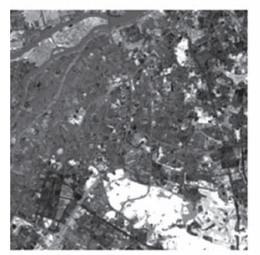

(d)

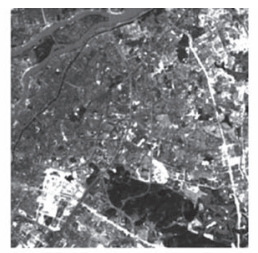

(b)

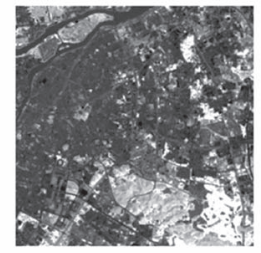

(e)

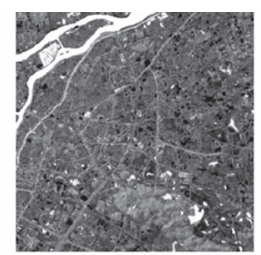

(c)

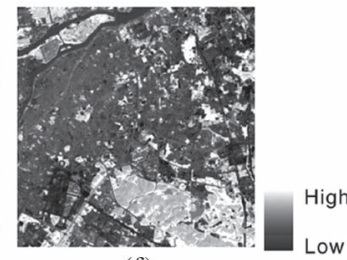

(f)
Fig. 13. Class proportion images obtained by using LSMM.

those produced by the MLA methods. This is because of the heterogeneous spectral signatures for the TM data. However, LSMM and MLA were both successful in identifying water, urban, and developing land classes.

Although soft classification (with proportions for each class within a pixel) can be generated, a hardening process is sometimes required to obtain final crisp assignments to each class (with only one single land class for a pixel) in applications [38]. This is done by applying appropriate ranking procedures and decision rules based on the inherent uncertainty and total amount of information dormant within the data. The hardening rule in this paper is represented as follows:

$$
\operatorname{class}(i) \leftarrow \arg \max \left(p^{c}(i)\right)
$$

where $p^{c}(i)$ is the proportion of the $c$ th land use.

Classification results by hardening the soft outputs from MLA are shown in Fig. 14(a). The hardening results from this MLA method are compared with those from the artificial neural networks (ANN), the maximum likelihood (ML), and the minimum distance method. The classification results of these approaches are shown in Fig. 14(b)-(d), respectively. As shown in Fig. 14, MLA achieved the most satisfactory results since each land-use class can be effectively identified. ANN also yields good classification results for most classes but gives unsatisfactory or even incorrect results for the classification of grass and forest (listed in red frame). The ML approach magnifies the area of the developing land and misidentifies a road as a water object [Fig. 14(c)]. As shown in Fig. 14(d), the minimum distance method exhibits the worst performance in the classification of grass, cropland, urban, and developing land (listed in red frame).

An error matrix is used to calculate the overall accuracy of the hard classification. Using an error matrix to represent accuracy has been adopted by many researchers as it provides a detailed assessment of the agreement between the sample reference data and classification data at specific locations [38]. As shown in Table IV, the overall accuracy is $91.6 \%$ when using this MLA model. All land-use classes in the study area have been identified successfully. In contrast, ANN, ML, and the minimum distance classifiers have obtained lower overall accuracies. However, the overall accuracy has a bias because

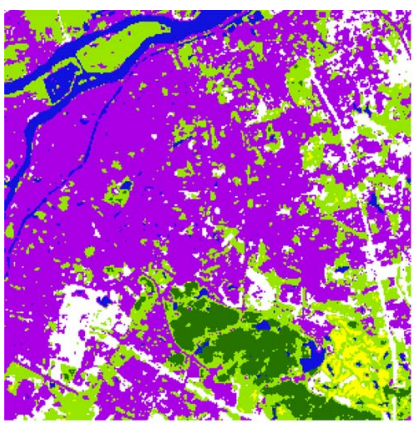

(a)

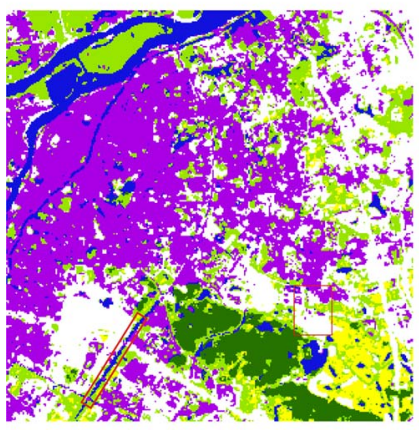

(c)

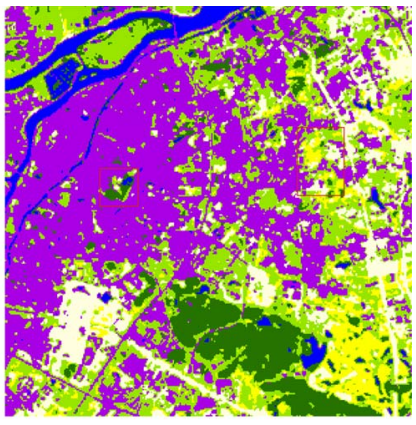

(b)

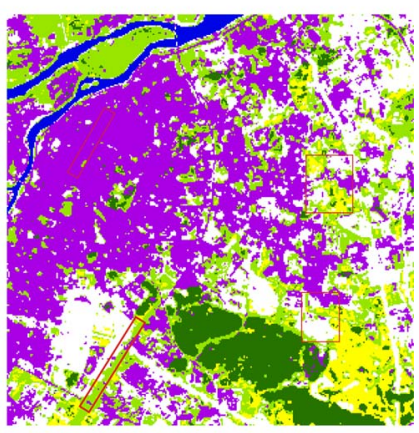

(d)

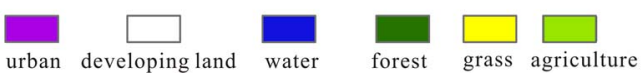

Fig. 14. Land-use classification in the study area of Dongguan.

of the difference between the actual and chance agreements, which can be effectively explained with the kappa coefficient [5]. As a result, more meaningful results will be yielded by using the kappa coefficient in assessing remote-sensing classification. The kappa coefficients of MLA, ANN, ML, and minimum distance are $0.903,0.867,0.832$, and 0.816 , respectively, (Table IV). This reveals that MLA is better than the other three traditional methods for land-cover classification.

\section{CONCLUSION}

Remote sensing is attractive in creating land-cover maps. However, when there are uncertainties in the form of mixed pixels in remote-sensing images, application of conventional "hard" classification methods to images dominated with mixed pixels may be very inappropriate and erroneous. In order to avoid this mixed-pixel problem, this paper has presented a new approach to improve the classification performance of remotesensing applications based on MLA. Being distinct from conventional classification approaches, this MLA model allows the creation of partial and multiple class memberships for mixed pixels and can calculate class proportions within a pixel so as to produce soft classification from remote-sensing data.

This MLA model is established by using a nonparametric regression algorithm, which is the k-NN. An NI approach is proposed to calculate the weights of $\mathrm{k}-\mathrm{NNs}$, using a greedy search strategy to search the optimal parameter settings for MLA. This model does not make any rigid assumption about the data, which relies on the data to determine a relationship between the input and output class proportions. In this paper, we 
TABLE IV

Comparison of the Classifications Obtained From MLA, Neural Network, ML, AND Minimum Distance

\begin{tabular}{ccccc}
\hline Classification method & MLA & neural network & maximum likelihood & minimum distance \\
\hline urban & 91.1 & 89.2 & 86.3 & 84.5 \\
forest & 92.2 & 88.7 & 90.5 & 88.6 \\
water & 96.8 & 97.8 & 91.3 & 88.2 \\
grass & 92.5 & 85.1 & 87.2 & 86.3 \\
agriculture & 88.4 & 85.5 & 84.2 & 81.4 \\
developing land & 93.7 & 92.6 & 81.5 & 81.9 \\
Overall accuracy (\%) & 91.6 & 88.7 & 85.3 & 83.7 \\
Kappa coefficient & 0.903 & 0.867 & 0.832 & 0.816 \\
\hline
\end{tabular}

have applied MLA to the soft classification of remote-sensing data. The results show that MLA is effective in inducing class proportions. MLA proves to be simple, flexible, and feasible and does not rely on the assumption regarding the relationship between spectral information and class proportions. The results of the classifications from a simulated image and a Landsat TM image clearly show that MLA produces a considerably higher accuracy of soft classification as compared with the conventional LSMM method. Therefore, MLA is a potentially useful approach in producing meaningful soft classifications from remote-sensing data.

The dominant class in a pixel is hardened by using MLA. MLA has an overall accuracy of $91.6 \%$ and a kappa coefficient of 0.903. ANN, ML, and the minimum distance methods have an overall accuracy of $88.7 \%, 85.3 \%$, and $83.7 \%$ and a kappa coefficient of $0.867,0.832$, and 0.816 , respectively. Therefore, this MLA model shows a higher classification accuracy than the other three approaches.

Compared with LSMM, MLA demonstrates some advantages. They mainly include the following: 1) MLA shows a higher classification accuracy than LSMM; 2) LSMM requires a priori knowledge of the signatures of endmembers present in images, which is generally not available [39]; 3) LSMM is based on an unattainable assumption that the class mixing is performed in a linear manner. However, MLA does not make any rigid assumption about the data [23]; 4) Moreover, LSMM cannot satisfy the requirement that each class proportion in the analysis result is within the range (0-1), while MLA can meet this requirement; and 5) Finally, LSMM assumption limits the number of primitive classes $c$, which can be identified by $c \leq n+1$, where $n$ is the number of measured spectral bands [40].

There are still some limitations in using this method in classifying remote-sensing data. First, the quality of the training data set used is a major determinant of the classification accuracy of MLA. These training sets are mainly obtained through visual interpretation of high-resolution satellite images or collection of ground data, which will spend considerable time and resources. In future researches, it is hoped that we can use small training sets for soft image classification by using semisupervised classification techniques. Lastly, the greedy search strategy is used to optimize parameter settings in this paper, while this approach takes a long time to achieve the optimization progress. Future work should be focused on searching the optimal parameter settings in a much shorter time by using artificial intelligence methods.

\section{REFERENCES}

[1] G. G. Wilkinson, "Results and implications of a study of fifteen years of satellite image classification experiments," IEEE Trans. Geosci. Remote Sens., vol. 43, no. 3, pp. 433-440, Mar. 2005.

[2] S. Di Zenzo, R. Bernstein, S. D. Degloria, and H. G. Kolsky, "Gaussian maximum likelihood and contextual classification algorithms for multicrop classification," IEEE Trans. Geosci. Remote Sens., vol. GRS-25, no. 6, pp. 805-814, Nov. 1987.

[3] K. Muthu and M. Petrou, "Landslide-hazard mapping using an expert system and a GIS," IEEE Trans. Geosci. Remote Sens., vol. 45, no. 2, pp. 522-531, Feb. 2007.

[4] F. Del Frate, F. Pacifici, G. Schiavon, and C. Solimini, "Use of neural networks for automatic classification from high-resolution images," IEEE Trans. Geosci. Remote Sens., vol. 45, no. 4, pp. 800-809, Apr. 2007.

[5] X. P. Liu, X. Li, and L. Liu, "An innovative method to classify remotesensing images using ant colony optimization," IEEE Trans. Geosci. Remote Sens., vol. 46, no. 12, pp. 4198-4208, Dec. 2008.

[6] G. M. Foody, "Fully fuzzy supervised classification of land cover from remotely sensed imagery with an artificial neural network," Neural Comput. Appl., vol. 5, no. 4, pp. 238-247, Dec. 1997.

[7] G. M. Foody, "Hard and soft classifications by a neural network with a non-exhaustively defined set of classes," Int. J. Remote Sens., vol. 23, no. 18 , pp. 3853-3864, Sep. 2002.

[8] J. B. Campbell, Introduction to Remote Sensing. New York: Guilford Press, 1987.

[9] T. F. Wood and G. M. Foody, "Using cover-type likelihoods and typicalities in a geographic information system data structure to map gradually changing environments," in In Landscape Ecology and GIS, R. HainesYoung, D. R. Green, and S. H. Cousins, Eds. London, U.K.: Taylor \& Francis, 1993, pp. 141-146.

[10] J. B. Campbell, Introduction to Remote Sensing., 3rd ed. New York: Guilford Press, 2002.

[11] A. C. Bernard, G. G. Wilkinson, and I. Kanellopoulos, "Training strategies for neural network soft classification of remotely-sensed imagery," Int. J. Remote Sens., vol. 18, no. 8, pp. 1851-1856, May 1997.

[12] M. A. Ibrahim, M. K. Arora, and S. K. Ghosh, "Estimating and accommodating uncertainty through the soft classification of remote sensing data," Int. J. Remote Sens., vol. 26, no. 14, pp. 2995-3007, Jul. 2005.

[13] A. Plaza, P. Martinez, R. Perez, and J. Plaza, "A quantitative and comparative analysis of endmember extraction algorithms from hyperspectral data," IEEE Trans. Geosci. Remote Sens., vol. 42, no. 3, pp. 650-663, Mar. 2004.

[14] C.-I Chang and B. Ji, "Weighted abundance-constrained linear spectral mixture analysis," IEEE Trans. Geosci. Remote Sens., vol. 44, no. 2, pp. 378-388, Feb. 2006.

[15] D. Lu and Q. Weng, "Use of impervious surface in urban land-use classification," Remote Sens. Environ., vol. 102, no. 1/2, pp. 146-160, May 2006.

[16] M. Xu, P. Watanachaturaporn, P. K. Varshney, and M. K. Arora, "Decision tree regression for soft classification of remote sensing data," Remote Sens. Environ., vol. 97, no. 3, pp. 322-336, Aug. 2005.

[17] G. M. Foody and A. Mathur, "The use of small training sets containing mixed pixels for accurate hard image classification: Training on mixed spectral responses for classification by a SVM," Remote Sens. Environ., vol. 103, no. 2, pp. 179-189, Jul. 2006.

[18] G. M. Foody, "Estimation of sub-pixel land cover composition in the presence of untrained classes," Comput. Geosci., vol. 26, no. 4, pp. 469478, May 2000

[19] J. Lein, "Toward the rapid characterization of the built environment within the wildland-urban interface: A soft classification strategy," GISci. Remote Sens., vol. 43, no. 1, pp. 44-61, Apr.-Jun. 2006. 
[20] M. R. Boutell, J. Luo, X. Shen, and C. M. Brown, "Learning multi-label scene classification," Pattern Recognit., vol. 37, no. 9, pp. 1757-1771, Sep. 2004.

[21] M.-L. Zhang and Z.-H. Zhou, "Multilabel neural networks with applications to functional genomics and text categorization," IEEE Trans. Knowl. Data Eng., vol. 18, no. 10, pp. 1338-1351, Oct. 2006.

[22] R. L. Kennedy, L. Yuchun, V. R. Benjamin, C. D. Reed, and R. P. Lippmann, Solving Data Mining Problems Through Pattern Recognition. Englewood Cliffs, NJ: Prentice-Hall, 1998.

[23] R. K. Oswald, W. T. Scherer, and B. L. Smith, "Traffic flow forecasting using approximate nearest neighbor nonparametric regression," Research Project Rep. for ITS Implementation Research 2000, Apr. 25, 2002. [Online]. Available: http://www.gmupolicy.net/its/papers.htm

[24] A. Nemes, W. J. Rawls, and Y. A. Pachepsky, "Use of a nonparametric nearest neighbor approach to estimate soil hydraulic properties," SSSA J., vol. 70, no. 2, pp. 327-336, 2006.

[25] U. Lall and A. Sharma, "A nearest-neighbor bootstrap for resampling hydrologic time series," Water Resour. Res., vol. 32, no. 3, pp. 679-693, 1996.

[26] F. Maselli, A. Rodolf, and C. Conese, "Fuzzy classification of spatially degraded thematic mapper data for the estimation of sub-pixel components," Int. J. Remote Sens., vol. 17, no. 3, pp. 537-551, Feb. 1996.

[27] F. Okeke and A. Karnieli, "Linear mixture model approach for selecting fuzzy exponent value in fuzzy c-means algorithm," Ecol. Inf., vol. 1, no. 1, pp. 117-124, Jan. 1996.

[28] R. G. Congalton, "A review of assessing the accuracy of classifications of remotely sensed data," Remote Sens. Environ., vol. 37, pp. 35-46, 1991.

[29] C. Small, "The Landsat ETM+ spectral mixing space," Remote Sens. Environ., vol. 93, no. 1/2, pp. 1-17, Oct. 2004.

[30] Q. H. Weng and X. F. Xu, "Medium spatial resolution satellite imagery for estimating and mapping urban impervious surfaces using LSMA and ANN," IEEE Trans. Geosci. Remote Sens., vol. 46, no. 8, pp. 2397-2406, Aug. 2008.

[31] Q. Du and C.-I Chang, "Linear mixture analysis-based compression for hyperspectral image analysis," IEEE Trans. Geosci. Remote Sens., vol. 42, no. 4, pp. 875-891, Apr. 2004.

[32] J. W. Boardman, F. A. Kruse, and R. O. Green, "Mapping target signatures via partial unmixing of AVIRIS data," in Proc. Summit JPL Airborne Earth Sci. Workshop, Pasadena, CA, 1995, pp. 23-26.

[33] M. E. Winter, "N-finder: An algorithm for fast autonomous spectral endmember determination in hyperspectral data," in Proc. Image Spectrom. $V$, 1999, vol. 3753, pp. 266-277.

[34] R. A. Neville, K. Staenz, T. Szeredi, J. Lefebvre, and P. Hauff, "Automatic endmember extraction from hyperspectral data for mineral exploration," in Proc. 4th Int. Airborne Remote Sens. Conf. Exhib., 1999, pp. 21-24.

[35] M. D. Craig, "Minimum-volume transforms for remotely sensed data," IEEE Trans. Geosci. Remote Sens., vol. 32, no. 3, pp. 542-552, May 1994.

[36] A. Ifarraguerri and C.-I Chang, "Hyperspectral image segmentation with convex cones," IEEE Trans. Geosci. Remote Sens., vol. 37, no. 2, pp. 756770, Mar. 1999.

[37] J. M. P. Nascimento and J. M. Dias, "Vertex component analysis: A fast algorithm to unmix hyperspectral data," IEEE Trans. Geosci. Remote Sens., vol. 43, no. 4, pp. 898-910, Apr. 2005.

[38] E. Binaghi, P. A. Brivio, P. Ghezzi, and A. Rampini, "A fuzzy set based accuracy assessment of soft classification," Pattern Recognit. Lett., vol. 20, no. 9, pp. 935-948, Sep. 1999.

[39] D. C. Heinz and C.-I Chang, "Fully constrained least squares linear spectral mixture analysis method for material quantification in hyperspectral imagery," IEEE Trans. Geosci. Remote Sens., vol. 39, no. 3, pp. 529-545, Mar. 2001.

[40] M. Brown, H. G. Lewis, and S. R. Gunn, "Linear spectral mixture models and support vector machines for remote sensing," IEEE Trans. Geosci. Remote Sens., vol. 38, no. 5, pp. 2346-2360, Sep. 2000.

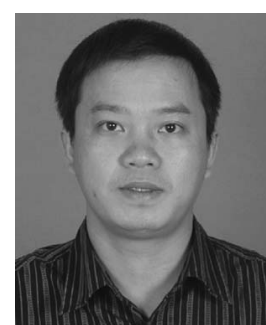

Xiaoping Liu received the B.S. degree in geography and the Ph.D. degree in remote sensing and geographical information sciences from Sun Yat-sen University, Guangzhou, China, in 2002 and 2008, respectively.

$\mathrm{He}$ is an Associate Professor with the School of Geography and Planning, Sun Yat-sen University. His current research interests include image processing, artificial intelligence, and geographical simulation.

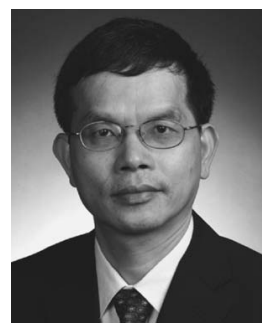

Xia Li received the B.S. and M.S. degrees from Peking University, Beijing, China, and the Ph.D. degree in geographical information sciences from the University of Hong Kong, Hong Kong.

$\mathrm{He}$ is a Professor and the Director of the Centre for Remote Sensing and Geographical Information Sciences, School of Geography and Planning, Sun Yat-sen University, Guangzhou, China. He is also a Guest Professor with the Department of Geography, University of Cincinnati, Cincinnati, OH. He is on the editorial board of the international journal Geojournal and is the Associated Editor of the Chinese journal Tropical Geography. He is the author of about 200 articles, of which many appeared in international journals. His papers are widely published in top international GIS and remote sensing journals, such as Remote Sensing of Environment, International Journal of Remote Sensing, Photogrammetric Engineering \& Remote Sensing, International Journal of Geographical Information Science, Environment and Planning A, and Environment and Planning B. His major research interests include remote sensing, geographical information systems for environmental modeling and land use planning, simulation of urban growth and land use changes with cellular automata and GIS, radar remote sensing for urban applications, and land use changes and agricultural land loss in the Pearl River Delta.

Dr. Li received the ChangJiang scholarship and the award of the Distinguished Youth Fund of the National Science Foundation of China in 2005.

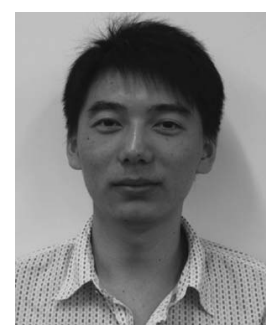

Xiaohu Zhang received the B.S. degree in geographical information sciences from Sun Yat-sen University, Guangzhou, China, in 2008. He is currently working toward the Master's degree with the University of Hong Kong, Hong Kong.

His main research areas include image processing and artificial intelligence. 